Bull. Austral. Math. Soc.

$11 \mathrm{M} 36,11 \mathrm{~F} 20,11 \mathrm{E} 45,11 \mathrm{H} 50,11 \mathrm{H} 31$

VOL. $62(2000)$ [243-251]

\title{
THE HEIGHT OF THE LEECH LATTICE
}

\author{
Kok Seng ChuA
}

\begin{abstract}
We derive explicit formulae for the height of the Leech lattice and give evidence supporting the conjecture that the Leech lattice has minimum height and minimum values of the Epstein zeta function among all 24 dimensional lattices of determinant one.
\end{abstract}

\section{INTRODUCTION AND RESULTS}

Let $(M, \sigma)$ be a compact Riemannian manifold with a smooth metric and let $0=\lambda_{0}<\lambda_{1} \leqslant \lambda_{2} \leqslant \ldots$ be the eigenvalues of the Laplacian. The height of $M$ is an isospectral invariant defined by $h(M, \sigma)=Z^{\prime}(0)$ where the right hand side is defined via the analytic continuation of the zeta function $Z(s)=\sum_{\lambda_{j} \neq 0} \lambda_{j}^{-s}$. In OsgoodPhillips-Sarnak [5], it was shown that when $M$ is the two dimensional torus, the height attains a minimum when and only when the metric is the flat metric corresponding to the hexagonal lattice which is the lattice with the highest sphere packing density. For 2 dimensional flat tori the height is given explicitly by Kronecker's limit formula in terms of the Dedekind eta function, see [5]. In [1], Chiu studied the heights of higher dimensional flat tori and proved the existence of a minimum in the moduli space. He also stated the following conjecture, attributed to Sarnak :

CONJECTURE 1. The height on the moduli space of $n$-dimensional flat tori of volume 1 has a global minimum at the torus corresponding to the lattice with the longest minimal vector (or equivalently the lattice giving the densest sphere packing).

Besides the two dimensional case, Conjecture 1 is further supported by the fact that the densest lattice in three dimensions, the face-centered cubic lattice is a local minimum for the height function and that its height is also strictly less than that of 10303 points uniformly distributed in the moduli space, see [1].

In this note, we shall give further evidence for Conjecture 1 in some higher dimensions. Most of our results can be proven in other dimensions but we shall concentrate on dimension 24 where there is a remarkable and very dense lattice (most likely the densest) discovered by Leech, and where our results are most explicit. Our main result

Received 22nd December, 1999

I would like to thank Yi Shan for inspiration.

Copyright Clearance Centre, Inc. Serial-fee code: 0004-9727/00 \$A2.00+0.00. 
will suggest that the Leech lattice is very likely the lattice with minimum height in dimension 24. We shall follow the notations and results in [1]. For a lattice specified by a positive definite quadratic form $\mathcal{Q}[x]$, we associate an Epstein zeta-function :

$$
Z_{\mathcal{Q}}(x)=\sum_{x \in Z^{n} \backslash\{0\}} \mathcal{Q}[x]^{-s}, \quad \operatorname{Re} s>n / 2 .
$$

This has a meromorphic continuation to the whole plane with a pole at $n / 2$ with residue $|\mathcal{Q}|^{-1 / 2} \pi^{n / 2} \Gamma(n / 2)^{-1}$. It was shown in [1, Theorem 2.3] that the height is given by $h(\mathcal{Q})=A_{n} k_{n}(\mathcal{Q})+B_{n}$ where $A_{n}, B_{n}$ are absolute constants with $A_{n}$ positive and the Kronecker limit of $\mathcal{Q}, k_{n}(\mathcal{Q})$ is defined by

$$
k_{n}(\mathcal{Q})=\lim _{s \rightarrow n / 2}\left\{Z_{\mathcal{Q}}(s)-\frac{|\mathcal{Q}|^{-1 / 2} \pi^{n / 2} \Gamma(n / 2)^{-1}}{s-n / 2}\right\} .
$$

Obviously instead of height we can work with $k_{n}(\mathcal{Q})$. We note incidentally that

$$
k_{n}(t \mathcal{Q})=\frac{1}{t^{n / 2}}\left\{k_{n}(\mathcal{Q})-\ln (t)|\mathcal{Q}|^{-1 / 2} \pi^{n / 2} \Gamma(n / 2)^{-1}\right\}
$$

so that we may compare height on any constant determinant surface. Our main result is the following:

Theorem 1. Let $\Lambda$ be the Leech lattice and $\mathcal{Q}$ be a 24 dimensional lattice of determinant 1. Then we have $k_{n}(\Lambda) \leqslant k_{n}(\mathcal{Q})$ in each of the following cases :

(A) $\mathcal{Q}$ is a 24 dimensional integral unimodular lattice.

(B) $\mathcal{Q}$ has a representation as a quadratic form which is diagonal.

(C) $\mathcal{Q}$ has minimum norm $m_{\mathcal{Q}}=\operatorname{Min}_{x \in Z^{24} \backslash\{0\}} \mathcal{Q}[x]$ bounded above by 1.6 or $m_{\mathcal{Q}^{-1}} \leqslant 0.38$.

We note that there are 297 unimodular lattices in dimension 24, and of these, 24 of them including the Leech lattice are even or type II, see [2]. The condition (A) is reassuring because in an extremal problem, there is a tendency for the extremal solution to be highly symmetric as the constraints that are applied then at various parts are equal. The unimodular lattices and in particular the Leech lattice are well known to have large automorphism groups.

As stated in Theorem 1, (B) is clearly implied by (C) but our method of proof of (A) and (B) actually proves more. In [1], Chiu stated a stronger conjecture attributed to Rankin:

CONJECTURE 2. Let $\mathcal{Q}$ be an $n$ dimensional lattice of determinant 1 and let $\mathcal{Q}_{0}$ be one with the highest sphere packing density (assume unique). Then for $s$ real and $s>0$, we have

$$
Z_{\mathcal{Q}}(s)-Z_{\mathcal{Q}_{0}}(s) \leqslant 0
$$


and equality holds for some $s$ if and only if $\mathcal{Q}$ is isometric to $\mathcal{Q}_{0}$.

We note that Conjecture 1 is equivalent to Conjecture 2 at $s=n / 2$ where (4) remains meaningful because the poles cancel out. Conjecture 2 is known to hold in dimension 2 and it is also known that (4) is true for sufficiently large $s$, see [6]. Mongomery [4] has established Conjecture 2 in dimension 2 by proving a yet stronger inequality, namely that the theta series of $\mathcal{Q}$ dominates that of the hexagonal lattice which trivially implies (4), see Lemmas 1 and 5 below. We shall prove in Section 2 that the theta series of a 24 dimensional $\mathcal{Q}$ dominates that of the Leech lattice under conditions (A) and (B), which implies the following :

THEOREM 2. Under the assumptions of Theorem 1 , we have $Z_{\mathcal{Q}}(s)-Z_{\Lambda}(s) \geqslant 0$ for all $s>0$ if either $(A)$ or $(B)$ holds.

We note that (A) or (B) of Theorem 1 is an immediate consequence of Theorem 2. Our proof of (C) however, works only for the height. Since the height becomes unbounded as $\mathcal{Q}$ tends to the boundary of the moduli space, that is, as $m_{\mathcal{Q}}$ tends to zero, one obtains (C) easily if one can compute the height of the Leech lattice explicitly. In fact, by using the well known correspondence between modular forms and Dirichlet series, it is easy to compute the heights of all the 24 even unimodular 24 dimensional lattices. Since there are five isospectral pairs among them, there are only 19 distinct theta series, see [2, Table 16.1]. These are parametrised by their Coxeter number $h$ defined by $24 h=$ number of vectors of norm 2 , so that $h=0$ corresponds to the Leech Lattice. Our formula for the height is :

ThEOREM 3. Let $\Gamma$ be an even unimodular 24 dimensional lattice with Coxeter number $h$. Then we have

$$
k_{n}(\Gamma)=\frac{\pi^{12}}{\Gamma(12)}\left\{\gamma-\ln (2)+\frac{\zeta^{\prime}}{\zeta}(12)-\frac{1}{\zeta(12)} \sum_{n=1}^{\infty} \frac{\tau(n)}{n^{12}}\right\}+\frac{24 h}{2^{12}} \sum_{n=1}^{\infty} \frac{\tau(n)}{n^{12}}
$$

where $\gamma$ is Euler's constant and $\tau(n)$ is the Ramanujan function. In particular for the Leech lattice, we have $k_{n}(\Lambda)=-0.025711 \ldots$.

In Section 3 we shall also derive an explicit formula for the height of an arbitrary $n$ dimensional lattice as a series of incomplete gamma functions. This gives in principle a way to compute the height of any lattice to any accuracy as long as its theta series (that is, the number of lattice vectors of each possible norms) is known. The formula is stated only in the case when $n$ is even since it is simpler and we only need it in this case:

ThEOREM 4. Let $\mathcal{Q}$ be an $n$ dimensional lattice of determinant one and assume 
$n$ is even. Let $G(m, a)=\int_{1}^{\infty} e^{-a t} t^{m}(d t / t)$ for integral $m$ and any positive $a$. Then

(6) $k_{n}(\mathcal{Q})=\frac{\pi^{n / 2}}{\Gamma(n / 2)}\left\{\ln (\pi)+\gamma-\sum_{j=1}^{n / 2} \frac{1}{j}+\sum_{a \in Z^{n}}^{\prime} G(n / 2, \pi \mathcal{Q}[a])+\sum_{a \in Z^{n}}^{\prime} G\left(0, \pi \mathcal{Q}^{-1}[a]\right)\right\}$

where $\gamma=0.577215665 \ldots$ is Euler's constant and the prime in the summation over a means the zero vector is omitted.

\section{ThETA SERIES DOMINANCE}

For a lattice $\Gamma$, its theta series is defined by

$$
\theta_{\Gamma}(z)=\sum_{\lambda \in \Gamma} q^{\langle\lambda, \lambda)}, \text { where } q=e^{\pi i z}
$$

For two lattices $\Gamma_{1}, \Gamma_{2}$ with the same determinant, we say that $\theta_{\Gamma_{1}}$, dominates $\theta_{\Gamma_{2}}$ and write $\theta_{\Gamma_{1}} \geqslant \theta_{\Gamma_{2}}$ if we have $\theta_{\Gamma_{1}}(i y) \geqslant \theta_{\Gamma_{2}}(i y)$ for all $y>0$. The relevance of this definition is given by the following lemma which is an observation of Montgomery in [4].

LEMMA 1. Let $\theta_{\Gamma_{1}} \geqslant \theta_{\Gamma_{2}}$, then $Z_{\Gamma_{1}}(s)-Z_{\Gamma_{2}}(s) \geqslant 0$ for all $s>0$.

PRoOF: By the old technique of Riemann, one can express $Z_{\Gamma}(s)$ as an integral over the theta series. More exactly, see [7, Theorem 2], we have

$$
\begin{aligned}
\pi^{-s} \Gamma(s) Z_{\Gamma}(s)= & \frac{|\Gamma|^{-1 / 2}}{s-n / 2}-\frac{1}{s} \\
& \quad+\int_{1}^{\infty}\left[\theta_{\Gamma}(i t)-1\right] t^{s} \frac{d t}{t}+|\Gamma|^{-1 / 2} \int_{1}^{\infty}\left[\theta_{\Gamma^{-1}}(i t)-1\right] t^{n / 2-s} \frac{d t}{t}
\end{aligned}
$$

Also the Jacobi inversion formula $\theta_{\Gamma}(i / t)=t^{n / 2} \theta_{\Gamma}(i t)$ implies that $\theta_{\Gamma_{1}}$ dominates $\theta_{\Gamma_{2}}$ if and only if $\theta_{\Gamma_{1}^{-1}}$ dominates $\theta_{\Gamma_{2}^{-1}}$ so that the claim follows immediately from (8).

Now let $\Gamma$ be any unimodular 24 dimensional lattice. By a classical theorem of Hecke see for example [3], $\theta_{\Gamma}(z)$ is a modular form of weight 12 for the Hecke subgroup $G(2)$ generated by $S=\left(\begin{array}{cc}0 & -1 \\ 1 & 0\end{array}\right)$ and $T^{2}=\left(\begin{array}{ll}1 & 2 \\ 0 & 1\end{array}\right)$ with respect to the character $\chi\left(T^{2}\right)=1$ and $\chi(S)=i$. Let $\theta_{3}(z)=\theta_{Z}(z)=\sum q^{m^{2}}$ be the series of the integer and let $\Delta_{8}=\left\{\theta_{3}^{8}-\theta_{E 8}\right\} / 16=q \prod_{m=1}^{\infty}\left\{\left(1-q^{2 m-1}\right)\left(1-q^{4 m}\right)\right\}^{8}$, the cusp form of weight 4, $E 8$ here being the root lattice giving the densest packing in dimension 8 . The space of modular form of weight 12 for $G(2)$ is of dimension 4 and is generated by $\left\{f_{j}=\theta_{3}^{24-8 j} \Delta_{8}^{j}: j=0,1,2,3\right\}$, see $[2, \mathrm{p} .87]$. 
LEMMA 2. $\theta_{E 8} \leqslant \theta_{3}^{8} \leqslant 2 \theta_{E 8}$.

PRoof: Following notation in [2], we have $\theta_{E 8}=\left\{\theta_{2}^{8}+\theta_{3}^{8}+\theta_{4}^{8}\right\} / 2$, so $2 \theta_{E 8}-\theta_{3}^{8}=$ $\theta_{2}^{8}+\theta_{4}^{8}$. Also $\theta_{3}^{8}-\theta_{E 8}=\left\{\theta_{3}^{8}-\left(\theta_{2}^{8}+\theta_{4}^{8}\right)\right\} / 2=\theta_{2}^{4} \theta_{4}^{4}$, using the well known formula of Jacobi that $\theta_{2}^{4}+\theta_{4}^{4}=\theta_{3}^{4}$. The result now follows from the fact that $\theta_{2}^{4}(i y)>0$ and $\theta_{4}^{4}(i y)>0$, which is obvious from their infinite product expansion, see [2, p.105].

LEMMA 3. $\theta_{3}^{8} \geqslant 32 \Delta_{8} \geqslant 0$ and hence $f_{1} \geqslant 32 f_{2}, f_{1} \geqslant 1024 f_{3}$.

PROOF: The first assertion follows from Lemma 2 and the definition of $\Delta_{8}$. It follows that $f_{1} \theta_{3}^{16} \Delta_{8} \geqslant 32 \theta_{3}^{8} \Delta_{8}^{2}=32 f_{2}$. Similarly, $f_{1} \geqslant 32^{2} f_{3}$.

We note incidentally that $f_{1}=\sum(-1)^{n+1} \tau(n) q^{n}$ and $f_{2}=\Delta=\sum \tau(n) q^{2 n}$ where $\tau(n)$ is the Ramanujan function. Both of these can be proven easily by looking at the infinite product expansions. Now let $\Lambda$ be the Leech lattice, then $\theta_{\Lambda}-\theta_{\Gamma}$ is a cusp form since both series have constant term one. We have in fact

Lemma 4. Suppose $\Gamma$ has $\lambda_{j}$ vectors of norm $j$ for $j=1,2,3$. Then

$$
\theta_{\Gamma}=\theta_{\Lambda}+\lambda_{1} f_{1}+\left(\lambda_{2}-24 \lambda_{1}\right) f_{2}+\left(\lambda_{3}-252 \lambda_{1}\right) f_{3} .
$$

ProOF: Since the Leech lattice has no vector of norm 1,2 or 3 , we must have $\theta_{\Gamma}=\theta_{\Lambda}+\lambda_{1} q+\lambda_{2} q^{2}+\lambda_{3} q^{3}+O\left(q^{4}\right)$. Now the space of cusp form for $G(2)$ has basis $\left\{f_{1}, f_{2}, f_{3}\right\}$ with initial expansions:

$$
\begin{aligned}
& f_{1}=\theta_{3}^{16} \Delta_{8}=q+24 q^{2}+252 q^{3}+O\left(q^{4}\right) \\
& f_{2}=\theta_{3}^{8} \Delta_{8}^{2}=q^{2}+O\left(q^{4}\right) \\
& f_{3}=\Delta_{8}^{3}=q^{3}+O\left(q^{4}\right) .
\end{aligned}
$$

It follows that $\left\{g_{1}, g_{2}, g_{3}\right\}$ with $g_{1}=f_{1}-24 f_{2}-252 f_{3}, g_{2}=f_{2}, g_{3}=f_{3}$ is another basis with $g_{i}$ having $j$ th Fourier coefficient $=\delta_{i j}$ for $1 \leqslant i, j \leqslant 3$. It follows that $\theta_{\Gamma}-\theta_{\Lambda}=\lambda_{1} g_{1}+\lambda_{2} g_{2}+\lambda_{3} g_{3}$ from which the result follows.

LEMMA 5. $\theta_{\Lambda} \leqslant \theta_{\Gamma}$.

Proof: We split $f_{1}=(3 / 4) f_{1}+(1 / 4) f_{1} \geqslant(3 / 4) 32 f_{2}+(1 / 4) 1024 f_{3}$ by Lemma 3. So by Lemma 4 we have $\theta_{\Gamma}-\theta_{\Lambda} \geqslant \lambda_{2} f_{2}+\left(\lambda_{3}+4 \lambda_{1}\right) f_{3}$. It follows from the infinite product expansion of $\theta_{3}$ and $\Delta_{8}$ that $f_{2}(i y)>0, f_{3}(i y)>0$. The Lemma follows since each $\lambda_{j} \geqslant 0$.

LemMa 6. (Montgomery [4, Theorem 2].) If $\mathcal{Q}$ is an $n$ dimensional determinant 1 lattice with a representation by a diagonal matrix, then $\theta_{Z}^{n} \leqslant \theta_{\mathcal{Q}}$.

Proof of Theorem 2: (A) follows immediately from Lemma 5 and Lemma 1. Since $Z^{24}$ is unimodular, (A) and Lemma 6 imply $\theta_{\Lambda} \leqslant \theta_{Z}^{24} \leqslant \theta_{\mathcal{Q}}$. So (B) holds by Lemma 1. 


\section{EXPLICIT FORMULAE FOR HEIGHTS}

We shall first derive explicit formulae for the heights of all the 24 even unimodular Niereimer lattices. An old result of Hecke implies that the $\theta$ series of such a lattice is a modular form of weight 12 for $S L_{2}(Z)$, which is of dimension 2 and is spanned by

$$
E_{12}=1+A_{12} \sum_{n=1}^{\infty} \sigma_{11}(n) q^{2 n}, A_{12}=\frac{(2 \pi)^{12}}{\Gamma(12) \zeta(12)}
$$

and the unique cusp form of weight 12 ,

$$
\Delta(z)=\sum_{n=1}^{\infty} \tau(n) q^{2 n}
$$

LEMMA 7 . For the Leech lattice $\Lambda$,

$$
Z_{\Lambda}(s)=\frac{A_{12}}{2^{s}}\left\{\zeta(s) \zeta(s-11)-\sum_{n=1}^{\infty} \frac{\tau(n)}{n^{s}}\right\}
$$

Proof: Since the Leech lattice has no vector of norm 2 and the cusp form is one dimensional, its theta series must be $\theta_{\Lambda}=E_{12}-A_{12} \Delta=1+A_{12} \sum_{n=1}^{\infty}\left[\sigma_{11}(n)-\right.$ $\tau(n)] q^{2 n}$. It follows that $Z_{\Lambda}(s)=A_{12} \sum_{n=1}^{\infty}\left(\sigma_{11}(n)-\tau(n)\right) /(2 n)^{s}$ and the result follows immediately.

We can now compute the height of the Leech lattice exactly.

LEMMA 8.

$$
k_{n}(\Lambda)=\frac{\pi^{12}}{\Gamma(12)}\left\{\gamma-\ln (2)+\frac{\zeta^{\prime}}{\zeta}(12)-\frac{1}{\zeta(12)} \sum_{n=1}^{\infty} \frac{\tau(n)}{n^{12}}\right\}
$$

Proof: Since $\zeta(s)=1 /(s-1)+\gamma+O(s-1)$, we have

$$
\zeta(s) \zeta(s-11)=\zeta(12) /(s-12)+\left[\zeta^{\prime}(12)+\gamma \zeta(12)\right]+O(s-12)^{2} .
$$

Also, $2^{-s}=2^{-12}-\left(\ln (2) / 2^{12}\right)(s-12)+O(s-12)^{2}$, so we must have

$$
\begin{aligned}
\left(A_{12} / 2^{s}\right) \zeta(s) \zeta(s-11) & =\frac{A_{12} \zeta(12) 2^{-12}}{s-12}+\frac{A_{12}}{2^{12}}\left\{\zeta^{\prime}(12)+\gamma \zeta(12)-\ln (2) \zeta(12)\right\} \\
& =\frac{\pi^{12} / \Gamma(12)}{s-12}+\frac{\pi^{12}}{\Gamma(12)}\left\{\frac{\zeta^{\prime}}{\zeta}(12)+\gamma-\ln (2)\right\} .
\end{aligned}
$$


The result follows from Lemma 7 since $\sum \tau(n) / n^{s}$ is regular at $s=12$.

LEMma 9. If $\Gamma$ is an even unimodular 24 dimensional lattice with Coxeter number $h$ (that is, if $\Gamma$ has $24 h$ vectors of norm 2 ), $\theta_{\Gamma}=\theta_{\Lambda}+24 h \Delta$.

Proof: The difference of the two theta series is a cusp form with leading term $24 h q^{2}$.

Proof of Theorem 3: By Lemma 9, $Z_{\Gamma}=Z_{\Lambda}+\left(24 h / 2^{s}\right) \sum\left(\tau(n) / n^{s}\right)$. Since the last term is regular at $s=12$, the result follows from Lemma 8 .

REMARK. We note that the series $\sum \tau(n) / n^{12}=0.994544 \ldots$ is easily computed as the series is absolutely convergent and we may estimate the error easily by Deligne's famous estimate that $|\tau(n)| \leqslant \sigma_{0}(n) n^{11 / 2}$.

We shall derive next an explicit formula for the height of an arbitrary lattice of determinant 1 (Theorem 4). We define first the integral (incomplete Gamma function): $G(m, a)=\int_{1}^{\infty} e^{-a t} t^{m} d t / t$ and note that for positive integral $m$, it can be evaluated by integration by parts and solving a recurrence.

LEMMA 10.

$$
G(m, a)=\frac{e^{-a}}{a}\left[1+\sum_{j=1}^{m-1} \frac{(m-1)(m-2) \ldots(m-j)}{a^{j}}\right]
$$

We also note that there is a continued fraction expansion of $G(m, a)$ which allows fast computation for any $m$ and $a$, see Terras [7].

Proof of Theorem 4: From (8) we have $Z_{\mathcal{Q}}(s)=\left(\pi^{s} / \Gamma(s)\right)\{1 /(s-n / 2)+$ $R(s)\}$ where $R(s)$ is regular at $n / 2$. Since

$$
\frac{\pi^{s}}{\Gamma(s)}=\frac{\pi^{n / 2}}{\Gamma(n / 2)}+\frac{\pi^{n / 2}}{\Gamma(n / 2)}\left\{\ln (\pi)-\frac{\Gamma^{\prime}}{\Gamma}(n / 2)\right\}(s-n / 2)+O(s-n / 2)^{2},
$$

we have

$$
\begin{aligned}
k_{n}(\mathcal{Q})= & \frac{\pi^{n / 2}}{\Gamma(n / 2)}\left\{\ln (\pi)-\frac{\Gamma^{\prime}}{\Gamma}(n / 2)+R(n / 2)\right\} \\
= & \frac{\pi^{n / 2}}{\Gamma(n / 2)}\left\{\ln (\pi)-\frac{\Gamma^{\prime}}{\Gamma}(n / 2)-\frac{2}{n}+\int_{1}^{\infty}\left[\theta_{\mathcal{Q}}(i t)-1\right] t^{n / 2} \frac{d t}{t}\right. \\
& \left.+\int_{1}^{\infty}\left[\theta_{\mathcal{Q}^{-1}}(i t)-1\right] \frac{d t}{t}\right\}
\end{aligned}
$$

Putting in $\left(\Gamma^{\prime} / \Gamma\right)(n / 2)=\sum_{j=1}^{n / 2-1} 1 / j-\gamma$ and writing out the sum over the lattice vectors gives (6). 
REMARK. Theorems 3 and 4 give us two distinct ways to compute the height of the Leech lattice and more generally its zeta function. By equating them we get a formula expressing the Ramanujan $L$-series in terms of a series of incomplete Gamma functions over lattice vectors which may be of some interest.

We are now ready to establish (C) of Theorem 1 . The idea is very simple. We can compute an explicit value of $k_{n}(\Lambda)$ by Theorem 3 while Theorem 4 allows us to bound it from below.

Proof of (C) of Theorem 1: Let $m_{\mathcal{Q}}, m_{\mathcal{Q}^{-1}}$ be the minimum norm of $\mathcal{Q}$ and $\mathcal{Q}^{-1}$ respectively. By Theorems 3 and 4 ,

$$
\begin{aligned}
k_{n}(\mathcal{Q})-k_{n}(\Gamma) \geqslant \frac{\pi^{12}}{\Gamma(12)}\{\ln (2 \pi) & -\frac{\zeta^{\prime}}{\zeta}(12)-\sum_{j=1}^{12} \frac{1}{j}+\frac{1}{\zeta(12)} \sum_{n=1}^{\infty} \frac{\tau(n)}{n^{12}} \\
& \left.+2 \sum_{k=1}^{\infty} G\left(12, \pi m_{\mathcal{Q}} k^{2}\right)+2 \sum_{k=1}^{\infty} G\left(0, \pi m_{\mathcal{Q}^{-1}} k^{2}\right)\right\}
\end{aligned}
$$

where we have ignored all lattice vectors of $\mathcal{Q}$ and $\mathcal{Q}^{-1}$ except for the multiples of a pair of shortest ones. Because $G\left(12, \pi m_{\mathcal{Q}} k^{2}\right)$ decreases exponentially in $k$, it does not really help to include higher multiples of the shortest vector. The first assertion follows simply from the fact that $G\left(12, \pi m_{\mathcal{Q}}\right)$ is decreasing in $m_{\mathcal{Q}}$ and that

$2 G(12,1.6 \pi)=0.305123 \ldots>\left\{\ln (2 \pi)-\frac{\zeta^{\prime}}{\zeta}(12)-\sum_{j=1}^{12} \frac{1}{j}+\frac{1}{\zeta(12)} \sum_{n=1}^{\infty} \frac{\tau(n)}{n^{12}}\right\}=0.27863 \ldots$

For the second assertion, setting $r=m_{\mathcal{Q}^{-1}}$, we see that we need

$$
\begin{aligned}
2 \sum_{k=1}^{\infty} G\left(0, \pi r k^{2}\right) & =2 \int_{l}^{\infty} \sum_{k=1}^{\infty} e^{-\pi r k^{2} t} \frac{d t}{t} \geqslant 2 \int_{1}^{T} \int_{1}^{\infty} e^{-\pi r t x^{2}} d x \frac{d t}{t} \text { for any } T>0, \\
& \geqslant 2 \int_{l}^{T} \int_{\sqrt{\pi r t}}^{\infty} e^{-u^{2}} \frac{d u}{\sqrt{\pi r t}} \frac{d t}{t} \geqslant \frac{4(1-1 / \sqrt{T})}{\sqrt{\pi r}} \int_{T}^{\infty} e^{-t^{2}} d t, \\
& \geqslant 0.27863,
\end{aligned}
$$

or equivalently

$$
m_{\mathcal{Q}^{-1}} \leqslant \frac{16}{\pi}\left\{\frac{(1-1 / \sqrt{T})}{0.270863} \int_{T}^{\infty} e^{-t^{2}} d t\right\}^{2}
$$

Using the very rough estimate $(1-1 / \sqrt{T}) \int_{T}^{\infty} e^{-t^{2}} d t \leqslant g(T)=(1-1 / T) e^{-T} \leqslant$ $g((1+\sqrt{5}) / 2)=0.075739 \ldots$ gives the required sufficient crude bound $r<0.381 \ldots . \square$ 
REMARK 1. Since the Leech lattice has minimal norm 4 and the minimal norm functional is continuous in moduli space, there ought to be lattices with norm arbitrary close to 4 near the Leech lattice. The first assertion of (C) is thus still some way off the truth. However, the proof of $(\mathrm{C})$ shows that further progress may be possible from better ways to estimate (6) from below by combining the estimate of $\mathcal{Q}$ and $\mathcal{Q}^{-1}$ and also possibly including the contributions of other lattice vectors which are not multiples of the shortest ones.

REMARK 2. It is clear that some or most of our results can be proven in similar way in other dimensions $n$ in particular when $n$ is divisible by 8 . We only mention the case $n=8$ when the result is even simpler and $E 8$ is known to be the densest lattice. The analogue of Theorem $1(\mathrm{~A})$ and $(\mathrm{B})$ and Theorem 2 holds. We also have $\theta_{E 8}=$ $E_{4}=1+240 \sum \sigma_{3}(n) q^{2 n}$ so that the zeta function $Z_{E 8}(s)=\left(240 / 2^{s}\right) \zeta(s) \zeta(s-3)$ and $k_{n}(E 8)=\left(\pi^{4} / \Gamma(4)\right)\left\{\zeta^{\prime} / \zeta(4)+\gamma-\ln (2)\right\}$. A similar argument now shows that for an 8 dimensional lattice of determinant $1, k_{n}(E 8) \leqslant k_{n}(\mathcal{Q})$ if $m_{\mathcal{Q}} \leqslant 0.8$ or $m_{\mathcal{Q}^{-1}} \leqslant 0.49$. Note that the minimal norm of $E 8$ is 2 .

\section{REFERENCES}

[1] P. Chiu, 'Height of flat tori', Proc. Amer. Math. Soc. 125 (1997), 723-730.

[2] J. Conway and J. Sloane, Sphere packings, lattices and groups, (second edition) (SpringerVerlag, Berlin, Heidelberg, New York, 1988).

[3] E. Hecke, 'Lectures on Dirichlet series, modular functions and quadratic forms', (1983).

[4] H. Montgomery, 'Minimal theta functions', Glasgow Math. J. 30 (1988), 75-85.

[5] B. Osgood, R. Phillips and P. Sarnak, 'Extremals of determinants of Laplacians', J. Funct. Anal. 80 (1988), 148-211.

[6] S. Ryshkov, 'On the equation of final $\zeta$-optimality of lattices providing the densest lattice packing of $n$-dimensional sphere', (English translation), Siberian Math. J. 14 (1973), 743-750.

[7] A. Terras, "The minimum of quadratic form and the behaviour of Epstein and Dedekind zeta functions', J. Number Theory 12 (1980), 258-272.

Institute of High Performance Computing

89C Science Park Drive

\#02-11/12 The Rutherfold

Singapore 118261

e-mail: chuaks@ihpc.nus.edu.sg 